\title{
Neural representation of face familiarity in an awake chimpanzee
}

Evaluating the familiarity of faces is critical for social animals as it is the basis of individual recognition. In the present study, we examined how face familiarity is reflected in neural activities in our closest living relative, the chimpanzee. Skin-surface event-related brain potentials (ERPs) were measured while a fully awake chimpanzee observed photographs of familiar and unfamiliar chimpanzee faces (Experiment 1) and human faces (Experiment 2). The ERPs evoked by chimpanzee faces differentiated unfamiliar individuals from familiar ones around midline areas centered on vertex sites at approximately $200 \mathrm{~ms}$ after the stimulus onset. In addition, the ERP response to the image of the subject's own face did not significantly diverge from those evoked by familiar chimpanzees, suggesting that the subject's brain at a minimum remembered the image of her own face. The ERPs evoked by human faces were not influenced by the familiarity of target individuals. These results indicate that chimpanzee neural representations are more sensitive to the familiarity of conspecific than allospecific faces. 
1 Authors: Hirokata Fukushima ${ }^{1,2^{*}}$, Satoshi Hirata ${ }^{3}$, Goh Matsuda ${ }^{1,4}$, Ari Ueno ${ }^{1,5}$, Kohki Fuwa $^{6}$,

2 Keiko Sugama, Kiyo Kusunoki ${ }^{6}$, Kazuo Hiraki ${ }^{1}$, Masaki Tomonaga ${ }^{7}$, and Toshikazu Hasegawa ${ }^{1}$

$3 \quad{ }^{1}$ Graduate School of Arts and Sciences, The University of Tokyo, Japan

$4 \quad{ }^{2}$ Faculty of Sociology, Kansai University, Japan

$5 \quad{ }^{3}$ Wildlife Research Center, Kyoto University, Japan

$6 \quad{ }^{4}$ JST, CREST, Japan

$7 \quad{ }^{5}$ Department of Human Relations Studies, School of Human Cultures, The University of Shiga

8 Prefecture, Japan

$9 \quad{ }^{6}$ EarthMate-ChimpanzeeNEXT, Japan

$10 \quad{ }^{7}$ Section of Language and Intelligence, Primate Research Institute, Kyoto University, Japan

$11{ }^{*}$ Corresponding author:

12 Faculty of Sociology, Kansai University

13 3-3-35 Yamate-cho, Suita, Osaka 564-8680, Japan

14 Tel: +81-6-6368-0732

15 E-mail: fukush@kansai-u.ac.jp 


\section{Introduction}

As social animals, primates rely on face recognition to identify individuals and recognize

their emotional states. Until recently, vast knowledge of the primate visual system has been

primarily obtained from the brains of macaque monkeys and humans. Previous research has

suggested that the ventrolateral visual areas are central for face recognition both for monkeys and

humans (Perrett, Rolls, \& Caan, 1982; Tsao, Freiwald, Knutsen, Mandeville, \& Tootell, 2003).

However, the neural mechanisms responsible for face recognition in our closest living species,

the chimpanzee, remain relatively unknown. Examining these mechanism would shed new light

on the physiological and evolutionary origins of human social cognition. Behavioral studies of

chimpanzees have revealed that face stimuli attract their visual attention, just as in humans,

indicating the importance of face perception for chimpanzees (Kano \& Tomonaga, 2009). It has

been further reported that chimpanzees process face configurations in a similar manner to humans

(Hirata, Fuwa, Sugama, Kusunoki, \& Fujita, 2010; Parr, 2011; Tomonaga, 2007), they understand

conspecific facial expressions (Parr, 2003), and they have sophisticated abilities to identify individuals in terms of kinship detection (Parr \& de Waal, 1999). 
34 2009; Rilling et al., 2007; Taglialatela, Russell, Schaeffer, \& Hopkins, 2008, 2009; Ueno et al.,

35 2008, 2010). In particular, several research groups have reported neural examinations of

36 chimpanzee visual processing (Boysen \& Berntson, 1985; Fukushima et al., 2010; Parr et al.,

37 2009). For instance, Parr and colleagues (Parr et al., 2009) conducted a functional neuroimaging

38 study of chimpanzee face processing using positron emission tomography (PET). In their study,

39 the subject chimpanzees were given an isotope marker and performed a matching task with conspecific faces and objects, and they were then sedated before their brains were scanned. Their results explicitly revealed that the chimpanzees recruit similar neurocognitive substrates to humans for face perception. However, measurements of neural metabolism using methods such as PET imaging are poor metrics of the temporal aspects of the neural processing. Furthermore, non-invasive measurements of primates' brains often require sedation (Boysen \& Berntson, 1985; Parr et al., 2009; Rilling et al., 2007; Taglialatela et al., 2008, 2009), and this severely limits realtime measurement of their cognitive functions, particularly for elucidating socio-emotional responses.

49 in an adult female chimpanzee by means of scalp event-related potentials (ERPs) while she is

50 fully awake (Fukushima et al., 2010; Hirata et al., 2013; Ueno et al., 2008, 2010). Using this

51 protocol, we previously revealed neural evidence that this chimpanzee discriminates human faces 
52 from other categories of objects, as there was a divergence of ERPs in response to face stimuli at

53 approximately a $100 \mathrm{~ms}$ latency at posterior temporal regions (Fukushima et al., 2010).

54 Furthermore, we demonstrated that chimpanzee neural activities are influenced by observing

55 emotional expressions of conspecifics with an approximately 210 ms latency (Hirata et al., 2013).

56 Along this line of research, one issue to be further clarified is the influence of experience on

57 face processing. Particularly, familiarity is considered to be the basis of individual recognition

58 and an important factor in the modulation of social behaviors, by means of in-group and out-

59 group differentiation, for example. Human ERP studies show that a negative deflection around

60 140-200 latency ('N170') reflects a primal stage of face processing (Bentin, Allison, Puce, Perez,

$61 \&$ McCarthy, 1996; George, Evans, Fiori, Davidoff, \& Renault, 1996). Importantly, it has been

62 shown that the effects of facial familiarity appear in latencies later than $200 \mathrm{~ms}$ after stimulus

63 onset.That is, they appear later than the initial processing of basic facial configuration (Bentin \&

64 Deouell, 2000; Eimer, 2000; Henson et al., 2003; Schweinberger, Pfütze, \& Sommer, 1995).

65 Neuroimaging studies have further elucidated that facial familiarity modulates not only ventral

66 visual areas, but also distributed networks, including affective and memory-related regions,

67 including the amygdala and medial parietal areas (Gobbini \& Haxby, 2007; Natu \& O'Toole,

68 2011). Concerning chimpanzees, behavioral studies have revealed that familiarity with the target

69 enhances identification of individuals (Parr, Siebert, \& Taubert, 2011), but neural responses to 
familiarity during face perception remain unknown.

71 Based on these studies, the primary aim of the present study was to examine the effects of

72 familiarity on neural activities during face processing. The first experiment in this study

73 compared ERPs during the perception of familiar and unfamiliar conspecific faces. As a topic

74 somewhat related to familiarity perception, self-face recognition in chimpanzees is a research

75 topic that has received a great deal of attention (Bard, Todd, Bernier, Love, \& Leavens, 2006;

76 Gallup, 1970; Povinelli, 1987). The subject chimpanzee in the present study showed evidence of

77 mirror self-recognition, as indicated by self-exploratory behaviors of her own body while

78 watching a mirror (Hirata, unpublished data). Although the present study primarily examined the

79 effects of familiarity on face processing, the experiment also included an image of the subject's

80 own face as a stimulus category, as a first preliminary investigation of neural correlates of self-

81 perception in the chimpanzee.

82 It is also known that the species shown in a target stimulus can influence visual processing of

83 the displayed faces. However, whether or not primates have a specialized mechanism for

84 perceiving the faces of conspecifics is controversial (Martin-Malivel \& Okada, 2007; Parr,

85 Heintz, \& Pradhan, 2008). Among previous behavioral studies in chimpanzees, some findings

86 have suggested differential perception between conspecific and allospecific faces (Dahl,

87 Wallraven, Bulthoff, \& Logothetis, 2009; Fagot \& Tomonaga, 1999; Fujita, 1990; Hattori, Kano, 
88 \& Tomonaga, 2010; Parr, Dove, \& Hopkins, 1998; Parr, Heintz, \& Akamagwuna, 2006; Parr et

89 al., 2011; Tomonaga, Itakura, \& Matsuzawa, 1993), but some research has also suggested that

90 chimpanzees process human faces in a similar manner to conspecific faces (Kano \& Tomonaga,

91 2009; Martin-Malivel \& Okada, 2007; Tomonaga, 2007). These inconsistent findings from

92 behavioral studies motivate further elucidation of the species dependency of face processing by

93 identifying the neural responses to conspecific and non-conspecific faces and examining possible

94 differences in their patterns. Therefore, in the second experiment in this study, we presented the

95 subject with faces of allospecific humans, and compared the ERP responses with familiar and

96 unfamiliar human faces, to examine whether chimpanzee brain activity is differentially

97 responsive to the familiarity of conspecific and allospecific faces.

98 Materials and Methods

99 Subject

100 The subject was a female chimpanzee (Pan troglodytes) named Mizuki, who was housed at

101 the Great Ape Research Institute of Hayashibara Biochemical Laboratories, Inc., Okayama,

102 Japan. She was raised by human caregivers from a few days after birth. Since the subject arrived

103 at the Great Ape Research Institute when she was 2 years and 1 month old, she has spent the 
104

105

106

107

108

109

110

111

112

113

majority of her time with other chimpanzees in outdoor and indoor compounds. At the time of

experimentation, the subject was 10-11 years old and living in the institute with other group

members (two were male, two were female, and one was an infant). At that time, the subject had

undergone other behavioral cognitive experiments (Hirata \& Fuwa, 2007; Idani \& Hirata, 2006)

and earlier ERP experiments (Fukushima et al., 2010; Hirata et al., 2013; Ueno et al., 2008,

2010). She had also participated in informal tests of mirror self-recognition, recognition of self-

images on TV monitors, and match-to-sample tasks of the faces of conspecifics (Hirata,

unpublished data). A playground for the chimpanzees in the institute had a small wall covered

with stainless-iron, and it was observed that chimpanzees including the present subject looked at

the reflective wall and made some gestures, such as opening the mouth and looking into it.

This research was conducted in accordance with the "Guide for the Care and Use of

Laboratory Animals" of Hayashibara Biochemical Laboratories, Inc., and the Weatherall report,

"The use of non-human primates in research". The research protocol was approved by the Animal

Welfare and Animal Care Committee of The University of Tokyo and Hayashibara Biochemical

Laboratories, Inc. (GARI-051101).

Apparatus and stimuli

20 The overall methodology basically followed our previous study (Fukushima et al., 2010). The experimental room consisted of concrete walls, with moderate lighting. The subject sat on a 
122

123

124

125 126 display.

127

128

129

130

131

132

133

134 135

136

137

concrete platform and was fully awake during recordings. A 17-inch CRT display (IIyama

LA702U) was set up in front of her approximately $40 \mathrm{~cm}$ away, at the horizontal level of her

head. An infrared video camera was fixed on top of the CRT display to monitor the subject from a

frontal view. We used this camera to check if the subject's gaze was directed to the stimulus

Experimental stimuli in Experiment 1 were color photographs of the faces of chimpanzees

(Figure 1). The stimuli consisted of seven chimpanzee faces: three of unfamiliar (out-group)

chimpanzees that were novel to the subject, three of familiar (in-group) chimps, and the one of the subject's own face. The images were digitally processed in 24-bit color by graphics software.

The stimuli in Experiment 2 consisted of six human faces: three of familiar persons who were the caregivers of the subject, and three of unfamiliar humans who were novel to the subject. All of the human models were Japanese subjects in their 30s, and two male subjects and one female subject were included in both categories. In both experiments, all stimuli were displayed on a black background with a visual field approximately $15^{\circ}$ high and $13^{\circ}$ wide. The average luminance (mean, $119.29 \mathrm{~cd} / \mathrm{m}^{2} ; \mathrm{SD}, 0.73 \mathrm{~cd} / \mathrm{m}^{2}$ ) and number of pixels (mean, 94213; SD, 265) among stimulus categories were matched, varying less than $0.8 \%$.

138 Procedure 
140 five blocks with 105 trials (1575 trials in total). Experiment 2 was also conducted in three

141 recording sessions with different days from Experiment 1, each consisting of five blocks with 60

142 trials (900 trials in total). On each trial of both experiments, one of the stimuli (seven images in

143 Experiment 1 and six images in Experiment 2) was presented for $500 \mathrm{~ms}$ in a pseudo-randomized

144 order with no consecutive repetition of the same stimulus. Each stimulus was followed by a 700

$145 \mathrm{~ms}$ inter-stimulus interval (an empty black screen). To maintain the subject's attention to the

146 display, occasional images of several objects that were not related to faces or animals (e.g.,

147 geometric figures or patterns) were also presented randomly at a rate of once every several trials.

148 Between blocks, the subject was given a rest period of approximately $1 \mathrm{~min}$, which allowed her

149 to make considerable body movements and to receive fruit rewards. During the recordings, an

150 experimenter (one of the subject's caregivers) stood beside her to keep her still and facing the

151 display. The subject's gaze appeared to occasionally avert from the monitor. When this occurred,

152 another experimenter, who was monitoring the subject's gaze direction, manually added a marker

153 in the electroencephalography (EEG) data via a keyboard connected to the measurement

154 computer.

\section{ERP recording and analysis}

156 EEG was recorded from $\mathrm{Ag} / \mathrm{AgCl}$ electrodes attached to five scalp positions (Fz, $\mathrm{Cz}, \mathrm{Pz}, \mathrm{T}$, 
and T6), according to the international 10-20 system for humans. The signals were referenced to

158 the forehead midline $(\mathrm{FPz})$ and a ground electrode was positioned at the left earlobe (Fukushima et al., 2010). The electrodes were filled with Quick GEL and impedances were kept below $6 \mathrm{k} \Omega$. Signals were amplified by NuAmp-40 and processed by Acquire 4.3 software (NeuroScan Inc.) with a $1,000 \mathrm{~Hz}$ sampling rate. A $0.1-20 \mathrm{~Hz}$ band-pass filter $(24 \mathrm{~dB} /$ oct $)$ was applied in the offline analysis. All data were segmented into 700-ms epochs, including a 100-ms pre-stimulus baseline period, based on time markers of the stimulus onset. These epochs were baseline corrected with respect to the mean amplitude over the 100 -ms pre-stimulus period. Epochs that exceeded $\pm 60 \mu \mathrm{V}$ were excluded from the analysis. Epochs that contained 'non-looking' markers described above were also excluded in the analysis. The numbers of epochs accepted for the analysis were as follows: in Experiment 1, 115, 126, and 52, for unfamiliar, familiar, and self faces, respectively; in Experiment 2, 121 and 105 for unfamiliar and familiar faces, respectively. among the ERP amplitudes for stimulus categories. In Experiment 2, a successive two-tailed $t$ test was applied to explore the difference between ERPs to unfamiliar and familiar human faces.

173 To avoid the detection of spurious differentiation among categories, we considered a time range 174 of 30 consecutive time points $(30 \mathrm{~ms})$ of $p$-values $<0.05$ to indicate a significant main effect. 


\section{Results}

176

177

178

179

180

181

182 differentiation among the stimulus categories. A significant main effect of stimulus type was

The images of seven chimpanzee faces (three unfamiliar [out-group] chimpanzees, three familiar [in-group] chimps, and the subject herself; Figure 1) were pseudo-randomly presented during EEG measurements. ERPs time-locked to the presentation of the face stimuli were calculated for each category (i.e., unfamiliar/familiar/self).

The basic morphologies of the ERP waveforms remarkably resembled those reported in our previous studies with the same subject (Fukushima et al., 2010; Hirata et al., 2013). The ERPs from midline sites (Fz, Cz, and $\mathrm{Pz}$ ) showed three dominant deflections (Figure 2): early negative components within a $100 \mathrm{~ms}$ latency, positive deflections peaking around a 140-150 ms latency, and a negative slow wave, which was visible after approximately a $200 \mathrm{~ms}$ latency. At the lateral occipitotemporal sites (T5 and T6), additional negative components appeared over the time range of $100-150 \mathrm{~ms}$.

A successive analysis of variance (ANOVA) with a factor of stimulus type (unfamiliar/familiar/self) was applied along each ERP data point of each channel to test 191 detected over the midline electrode sites $(\mathrm{Fz}, \mathrm{Cz}$, and $\mathrm{Pz}$ ), as indicated in Figure 2 (all $F$-values $>$ 
$1923.027[d f 1=2, d f 2=290], p$-values $<0.05)$. This differentiation was found in the time range from

193 approximately a 200 to $450 \mathrm{~ms}$ latency, most prominently at the $\mathrm{Cz}$ site. The details of the

194 latencies that differentiated each category at each electrode position are depicted in Table 1 . Post-

195 hoc two-tailed $t$-test revealed that the waveforms for unfamiliar faces significantly diverged from

196 those of familiar and self faces and no differences in the ERPs related to familiar and self faces

197 were detected (Table 1).

198 Experiment 2

199 Following Experiment 1, in which we examined the effects of familiarity in ERP responses to

200 conspecific faces, Experiment 2 was conducted to investigate the same effect $\mathrm{s}$ of familiarity in

201 ERP responses to allospecific human stimuli. The images of six human faces (three persons

202 familiar and three persons unfamiliar to the subject) were pseudo-randomly presented using the

203 same basic design as in Experiment 1.

204 Figure 3 illustrates ERP waveforms elicited by the images of familiar and unfamiliar human

205 faces. A successive $t$-test $(d f=224)$ along each data point of the ERP waveforms on each channel

206 failed to detect any significant differences in the ERPs related to familiar and unfamiliar human

207 faces. 


\section{Discussion}

209 This study examined whether and how the familiarity of faces is reflected in chimpanzee

210 ERPs. Based on the results of Experiment 1, we propose that familiarity modulates the ERPs

211 evoked by conspecific faces, differentiating faces of familiar subjects from the subject's own

212 face. The familiarity effect was observed in a time range later than approximately $200 \mathrm{~ms}$ post-

213 stimulus onset. Our previous study reported that face-specific modulation of the chimpanzee's

214 visual ERP was initiated as early as around a $100 \mathrm{~ms}$ latency in comparison with images of

215 scrambled faces or non-face objects (Fukushima et al., 2010). Thus, the results of our previous

216 study and the current study together indicate an early response to face processing and relatively

217 late modulation by face familiarity. This pattern is consistent with humans studies, in which ERPs

218 differentiate familiar and unfamiliar faces in a time period after the primary processing of face

219 configuration (i.e., after the occurrence of the N170 component) (Bentin \& Deouell, 2000; Eimer,

220 2000; Henson et al., 2003; Kaufmann, Schweinberger, \& Burton, 2009; Miyakoshi, Kanayama,

221 Nomura, Iidaka, \& Ohira, 2008; Paller, Hutson, Miller, \& Boehm, 2003), although a few research

222 groups have reported earlier latencies of this familiarity effect (Caharel et al., 2002; Caharel,

223 Courtay, Bernard, Lalonde, \& Rebai, 2005; Jemel, Schuller, \& Goffaux, 2010; Leleu et al., 2010).

224 It is considered that the familiarity effect basically occurs in later stages of face recognition 
because it interacts with multiple face-related processing steps (Gobbini \& Haxby, 2007).

226 Interestingly, the latency of the familiarity effect in the present study resembled that of our

227 previous study of affective processing, in which the ERP differentiation of affective vs. non-

228 affective pictures initiated about a $210 \mathrm{~ms}$ latency (Hirata et al., 2013). Thus, the familiarity effect

229 in the current study may be partially modulated by affective processing.

230 With respect to its topography, the familiarity effect was observed around the midline

231 electrode sites (i.e., $\mathrm{Fz}, \mathrm{Cz}$, and $\mathrm{Pz}$ ) centered on the vertex. This result was different from our

232 previous study(Fukushima et al., 2010), which demonstrated face-specific patterns around

233 temporal and posterior regions (i.e., T5/T6 as well as Pz electrode sites), suggesting that the

234 familiarity effect in the current study was modulated by neural substrates not limited to those

235 specific to face processing. This consideration is again consistent with neuroimaging studies in

236 human subjects, which indicate that the familiarity effect is subserved by non-visual networks,

237 such as memory or affective networks (Gobbini \& Haxby, 2007; Natu \& O’Toole, 2011). Another

238 related study from our group examined the ERPs of the same subject while they received auditory

239 presentations of the names of familiar (including her own name) and unfamiliar chimpanzees,

240 showing that the evoked potentials from fronto-central sites differentiated unfamiliar names from

241 familiar ones (Ueno et al., 2010). Although the previous auditory study used different reference-

242 channels (i.e., A1/A2) from the current visual study, it is possible that similar mechanisms 
subserved familiarity processing across the two studies. Furthermore, a few human ERP studies

244 of face perception have reported a familiarity effect not only at temporo-occipital sites but also in

245 regions around fronto-central sites (Caharel et al., 2002; Miyakoshi et al., 2008). It is difficult to

246 strictly compare ERP topographies from chimpanzees and humans because of structural

247 differences in terms of the shapes of the bone and muscles of the skull between the species

248 (Burrows, Waller, Parr, \& Bonar, 2006), and these differences may substantially influence scalp-

249 surface potentials. However, the results of the present experiments are somewhat consistent with

250 those of previous human studies.

251 It is noteworthy that the ERPs elicited by the subject's own face diverged from those evoked by

unfamiliar but not familiar faces of conspecifics in Experiment 1. Human studies have shown that self-face perception is reflected in the modulation of face-evoked ERPs, although the latency and topography of this effect are somewhat inconsistent among reports (Caharel et al., 2002; Keyes,

Brady, Reilly, \& Foxe, 2010; Ninomiya, Onitsuka, Chen, Sato, \& Tashiro, 1998; Sui, Zhu, \&

Han, 2006). In our previous study mentioned above, the ERPs from the same subject chimpanzee showed specific patterns in their response to the auditory presentation of the subject's own name 
261 of exposure to this stimulus. Auditory symbols had been much more frequently used in the

262 subject's communication with human caregivers. As for the visual recognition of self-image, the

263 subject chimpanzee was exposed to the image of its own face in a few experimental sessions

264 (Hirata, unpublished), and she also had occasional access to visual images of her own face (see

265 Methods). However, the visual input of her own face may not be sufficient to differentiate ERPs

266 related to self and familiar non-self faces. This study at least suggests that experience with self-

267 face perception can influence neural representations of the self-face by shifting it toward that of

268 "familiar" individuals. In other words, we cannot claim based on our results that the subject

269 chimpanzee recognized an image of her own face as representing herself, but we can claim that

270 the subject's brain did respond differently to her own face. Determining whether there is a

271 chimpanzee neural signature of self-face recognition requires further investigation using

272 matching experience with the stimuli, and examination of the correspondence between neural

273 activities and measures of self-oriented behaviors.

274 In contrast to the responses to the chimpanzee faces, ERPs to human faces did not

275 differentiate familiar and unfamiliar individuals (Figure 2). This result suggests that modulation

276 of face familiarity in the chimpanzee visual system depends on the species displayed in the target

277 stimulus; that is, the chimpanzee was more sensitive to familiarity of conspecific faces than

278 allospecific faces. Behavioral studies in non-human primates have shown that monkeys have a 
relatively robust preference for conspecific faces despite substantial exposure to other species,

280 even when they lack visual experiences of conspecific faces (Fujita, 1993; Martin-Malivel \&

281 Okada, 2007; Sackett, 1966). Chimpanzees have also been shown to have self-species superiority

282 in social cognition, in terms of, for example, fast face recognition (Parr et al., 1998; Tomonaga et

283 al., 1993) and attracting initial looking at a conspecific face (Hattori et al., 2010). Therefore, the

284 differences between chimpanzee vs. human faces in the current study are in line with those

285 reports, and can be further assumed to suggest that visual face processing is an inherent property

286 of chimpanzees. However, in parallel to this view, a few studies have suggested the presence of

287 flexible modulation of cross-species face perception in chimpanzees. For example, captive

288 chimpanzees show similar configural processing to human faces as to conspecific faces (Parr et

al., 1998), and chimpanzees reared by humans show better discrimination of human faces than

chimpanzee faces (Martin-Malivel \& Okada, 2007). Furthermore, recent studies have explicitly

examined the effects of experience on cross-species face processing, showing that young

chimpanzees predominantly process conspecific faces, whereas old chimpanzees predominantly

process human faces (Dahl, Rasch, Tomonaga, \& Adachi, 2013a, 2013b). Their mathematical

simulations predicted that this shift in face processing occurs around the age of 15 years in 
298

299

300

301

302

303

304

305

306

307

308

309

310

311

312

313

humans, as it has been shown that human infants have a bias to conspecific faces (de Haan,

Pascalis, \& Johnson, 2002), but it may be also flexible as human adults perceive chimpanzee

faces with a quite similar process to that used for ose of human faces (Carmel \& Bentin, 2002).

Further investigations of face processing mechanism of primates should take into account

interactions between innate tendencies and acquired properties (de Haan, Humphreys, \& Johnson, 2002).

.Finally, we should be cautious of a couple of issues when interpreting the present findings.

First, it may be the case that neural differentiation of human familiarity did occur in Experiment 2

but it was so small that it was below the limit of detection of EEG or occurred in a manner that is

not amenable to measurement by cross-trial averaging of scalp-surface potentials (Luck, 2005;

Tallon-Baudry \& Bertrand, 1999). Therefore, it is still premature to conclude that the chimpanzee

is differentially sensitive to the conspecific and allospecific faces. Second, as pointed out in our

earlier discussion of the response to the self-face image, it is also possible that our results simply

reflect quantitative differences in the amount of exposure to the target categories. The present

subject was reared by humans, and interacted every day with the human staff whose faces were

presented as the stimuli of the "familiar" category in Experiment 2. Thus, we suggest that the

314 subject was very familiar with these human faces, and it is valid to define the set of pictures

315 depicting her caregivers as the "familiar" category for this subject. However, the amount of 
316 exposure to the familiar chimpanzees and humans, particularly the total amount of time

317 previously spent looking at their faces, could not be exactly and quantitatively matched in this

318 study. In common with the topic of self-face recognition, the current study was a preliminary

319 attempt to clarify the effects of familiarity on face processing and cross-species differences in

320 these effects. Further examinations of how experience modulates face perception should apply

321 strict control over exposure to the target stimuli and behavioral indices. 


\section{References}

323

324

325

326

327

328

329

330

331

332

333

334

335

Adey, W. R., Kado, R. T., \& Rhodes, J. M. (1963). Sleep: cortical and subcortical recordings in the chimpanzee. Science, 141, 932-933.

Bard, K. A., Todd, B., Bernier, C., Love, J., \& Leavens, D. (2006). Self-awareness in human and chimpanzee infants: what is measured and what is meant by the mark and mirror test? Infancy, 9(2), 191-219. Retrieved from http://sro.sussex.ac.uk/14481/

Bentin, S., Allison, T., Puce, A., Perez, E., \& McCarthy, G. (1996). Electrophysiological studies of face perception in humans. Journal of Cognitive Neuroscience, 8(6), 551-565. Retrieved from < Go to ISI >://A1996WF04200007

Bentin, S., \& Deouell, L. Y. (2000). Structural encoding and identification in face processing: erp evidence for separate mechanisms. Cognitive Neuropsychology, 17(1), 35-55. Retrieved from http://www.ncbi.nlm.nih.gov/pubmed/20945170

Boysen, S. T., \& Berntson, G. G. (1985). Visual evoked potentials in the great apes. Electroencephalography and Clinical Neurophysiology, 62(2), 150-153.

Burrows, A. M., Waller, B. M., Parr, L. A., \& Bonar, C. J. (2006). Muscles of facial expression in the chimpanzee (Pan troglodytes): descriptive, comparative and phylogenetic contexts. Journal of Anatomy, 208(2), $153-167$. doi:JOA523 [pii] 10.1111/j.1469-7580.2006.00523.x

Caharel, S., Courtay, N., Bernard, C., Lalonde, R., \& Rebai, M. (2005). Familiarity and emotional expression influence an early stage of face processing: an electrophysiological study. Brain and Cognition, 59(1), 96-100. doi:S0278-2626(05)00070-9 [pii] 10.1016/j.bandc.2005.05.005

Caharel, S., Poiroux, S., Bernard, C., Thibaut, F., Lalonde, R., \& Rebai, M. (2002). ERPs associated with familiarity and degree of familiarity during face recognition. International Journal of Neuroscience, 112(12), 1499-1512. doi:10.1080/00207450290158368

Carmel, D., \& Bentin, S. (2002). Domain specificity versus expertise: factors influencing distinct processing of faces. Cognition, 83(1), 1-29.

Dahl, C. D., Rasch, M. J., Tomonaga, M., \& Adachi, I. (2013a). Developmental processes in face perception. Scientific reports, 3, 1044. doi:10.1038/srep01044 
Dahl, C. D., Rasch, M. J., Tomonaga, M., \& Adachi, I. (2013b). The face inversion effect in non-human primates revisited - an investigation in chimpanzees (Pan troglodytes). Scientific reports, 3, 2504. doi:10.1038/srep02504

Dahl, C. D., Wallraven, C., Bulthoff, H. H., \& Logothetis, N. K. (2009). Humans and macaques employ similar faceprocessing strategies. Current Biology, 19(6), 509-513. doi:S0960-9822(09)00679-4 [pii] 10.1016/j.cub.2009.01.061

De Haan, M., Humphreys, K., \& Johnson, M. H. (2002). Developing a brain specialized for face perception: a converging methods approach. Developmental psychobiology, 40(3), 200-212.

De Haan, M., Pascalis, O., \& Johnson, M. H. (2002). Specialization of neural mechanisms underlying face recognition in human infants. Journal of Cognitive Neuroscience, 14(2), 199-209. doi:10.1162/089892902317236849 [doi]

Eimer, M. (2000). Event-related brain potentials distinguish processing stages involved in face perception and recognition. Clinical neurophysiology : official journal of the International Federation of Clinical Neurophysiology, 111(4), 694-705. doi:10.1016/S1388-2457(99)00285-0

Fagot, J., \& Tomonaga, M. (1999). Global and local processing in humans (Homo sapiens) and chimpanzees (Pan troglodytes): Use of a visual search task with compound stimuli. Journal of Comparative Psychology, 113(1), 3-12. Retrieved from $<$ Go to ISI $>$ ://000079202600001

Fujita, K. (1990). Species preference by infant macaques with controlled social experience. International Journal of Primatology, 11, 553-573.

Fujita, K. (1993). Development of visual preference for closely related】species by infant and juvenile macaques with restricted social experience. Primates, 34(2), 141-150. doi:10.1007/BF02381385

Fukushima, H., Hirata, S., Ueno, A., Matsuda, G., Fuwa, K., Sugama, K., Kusunoki, K., Hirai, M., Hiraki, K., Tomonaga, M., Hasegawa, T. (2010). Neural correlates of face and object perception in an awake chimpanzee (Pan troglodytes) examined by scalp-surface event-related potentials. PloS one, 5(10), e13366. doi:10.1371/journal.pone.0013366 
George, N., Evans, J., Fiori, N., Davidoff, J., \& Renault, B. (1996). Brain events related to normal and moderately scrambled faces. Brain Research. Cognitive Brain Research, 4(2), 65-76. doi:0926641095000453 [pii]

Gobbini, M. I., \& Haxby, J. V. (2007). Neural systems for recognition of familiar faces. Neuropsychologia, 45(1), 32-41. Retrieved from http://www.ncbi.nlm.nih.gov/pubmed/16797608

Hattori, Y., Kano, F., \& Tomonaga, M. (2010). Differential sensitivity to conspecific and allospecific cues in chimpanzees and humans: a comparative eye-tracking study. Biology Letters, 6(5), 610-613. Retrieved from http://www.pubmedcentral.nih.gov/articlerender.fcgi?artid=2936142\&tool=pmcentrez\&rendertype=abstract

Henson, R. N., Goshen-Gottstein, Y., Ganel, T., Otten, L. J., Quayle, A., \& Rugg, M. D. (2003). Electrophysiological and haemodynamic correlates of face perception, recognition and priming. Cerebral Cortex (Vol. 13, pp. 793805). Oxford Univ Press. Retrieved from http://www.ncbi.nlm.nih.gov/pubmed/12816895

Hirata, S., \& Fuwa, K. (2007). Chimpanzees (Pan troglodytes) learn to act with other individuals in a cooperative task. Primates, 48(1), 13-21. doi:10.1007/s10329-006-0022-1 [doi]

Hirata, S., Fuwa, K., Sugama, K., Kusunoki, K., \& Fujita, S. (2010). Facial perception of conspecifics: chimpanzees (Pan troglodytes) preferentially attend to proper orientation and open eyes. Animal Cognition, 13(5), 679-688. Retrieved from http://www.ncbi.nlm.nih.gov/pubmed/22466753

Hirata, S., Matsuda, G., Ueno, A., Fukushima, H., Fuwa, K., Sugama, K., Kusunoki, K., Tomonaga, M., Hiraki, K., Hasegawa, T. (2013). Brain response to affective pictures in the chimpanzee. Scientific reports, 3, 1342. doi:10.1038/srep01342

Idani, G., \& Hirata, S. (2006). Studies at the Great Ape Research Institute, Hayashibara. In D. A. Washburn (Ed.), Primate perspectives on behavior and cognition (pp. 29-36). Washington: American Psychological Association.

Jemel, B., Schuller, A.-M., \& Goffaux, V. (2010). Characterizing the spatio-temporal dynamics of the neural events occurring prior to and up to overt recognition of famous faces. Journal of Cognitive Neuroscience, 22(10), 2289-2305. Retrieved from http://www.ncbi.nlm.nih.gov/pubmed/19642891

Kano, F., \& Tomonaga, M. (2009). How chimpanzees look at pictures: a comparative eye-tracking study. Proceedings of the Royal Society of London, Series B: Biological Sciences, 276(1664), 1949-1955. doi:rspb.2008.1811 [pii] 10.1098/rspb.2008.1811 [doi] 
401

402

403

404

405

406

407

408

409

410

411

412

413

Kaufmann, J. M., Schweinberger, S. R., \& Burton, A. M. (2009). N250 ERP correlates of the acquisition of face representations across different images. Journal of Cognitive Neuroscience, 21(4), 625-641. Retrieved from http://eprints.gla.ac.uk/32221/

Keyes, H., Brady, N., Reilly, R. B., \& Foxe, J. J. (2010). My face or yours? Event-related potential correlates of selfface processing. Brain and Cognition, 72(2), 244-254. Retrieved from http://www.ncbi.nlm.nih.gov/pubmed/19854553

Leleu, A., Caharel, S., Carre, J., Montalan, B., Snoussi, M., Hofe, A. V, ... Rebai, M. (2010). Perceptual interactions between visual processing of facial familiarity and emotional expression: An event-related potentials study during task-switching. Neuroscience Letters, 482(2), 106-111. doi:10.1016/j.neulet.2010.07.008

Luck, S. (2005). An Introduction to the Event-Related Potential Technique. Cambridge MA: The MIT Press.

Martin-Malivel, J., \& Okada, K. (2007). Human and chimpanzee face recognition in chimpanzees (Pan troglodytes): role of exposure and impact on categorical perception. Behavioral Neuroscience, 121(6), 1145-1155. doi:200718058-001 [pii] 10.1037/0735-7044.121.6.1145

Miyakoshi, M., Kanayama, N., Nomura, M., Iidaka, T., \& Ohira, H. (2008). ERP study of viewpoint-independence in familiar-face recognition. International Journal of Psychophysiology, 69(2), 119-126. Retrieved from http://www.ncbi.nlm.nih.gov/pubmed/18474403

Natu, V., \& O’Toole, A. J. (2011). The neural processing of familiar and unfamiliar faces: A review and synopsis. British Journal of Psychology, 102(4), no-no. doi:10.1111/j.2044-8295.2011.02053.x

Ninomiya, H., Onitsuka, T., Chen, C. H. O., Sato, E., \& Tashiro, N. (1998). P300 in response to the subject's own face. Psychiatry and Clinical Neurosciences, 52(5), 519-522. doi:10.1046/j.1440-1819.1998.00445.x

Paller, K. A., Hutson, C. A., Miller, B. B., \& Boehm, S. G. (2003). Neural manifestations of memory with and without awareness. Neuron, 38(3), 507-516. Retrieved from http://www.ncbi.nlm.nih.gov/pubmed/12741996

Parr, L. A. (2003). The discrimination of faces and their emotional content by chimpanzees (Pan troglodytes). Annals of the New York Academy of Sciences, 1000, 56-78.

Parr, L. A. (2011). The evolution of face processing in primates. Philosophical Transactions of the Royal Society of London - Series B: Biological Sciences, 366(1571), 1764-1777. Retrieved from http://www.pubmedcentral.nih.gov/articlerender.fcgi?artid=3130377\&tool=pmcentrez\&rendertype=abstract 
Parr, L. A., \& de Waal, F. B. (1999). Visual kin recognition in chimpanzees. Nature, 399(6737), 647-648. doi:10.1038/21345 [doi]

Parr, L. A., Dove, T., \& Hopkins, W. D. (1998). Why faces may be special: evidence of the inversion effect in chimpanzees. Journal of Cognitive Neuroscience, 10(5), 615-622.

Parr, L. A., Hecht, E., Barks, S. K., Preuss, T. M., \& Votaw, J. R. (2009). Face processing in the chimpanzee brain. Current Biology, 19(1), 50-53. doi:S0960-9822(08)01566-2 [pii] 10.1016/j.cub.2008.11.048 [doi]

Parr, L. A., Heintz, M., \& Akamagwuna, U. (2006). Three studies on configural face processing by chimpanzees. Brain and Cognition, 62(1), 30-42. doi:S0278-2626(06)00071-6 [pii] 10.1016/j.bandc.2006.03.006

Parr, L. A., Heintz, M., \& Pradhan, G. (2008). Rhesus monkeys (Macaca mulatta) lack expertise in face processing. Journal of Comparative Psychology, 122(4), 390-402. doi:2008-16314-007 [pii] 10.1037/07357036.122.4.390

Parr, L. A., Siebert, E., \& Taubert, J. (2011). Effect of familiarity and viewpoint on face recognition in chimpanzees. Perception, 40(7), 863-872.

Perrett, D. I., Rolls, E. T., \& Caan, W. (1982). Visual neurones responsive to faces in the monkey temporal cortex. Experimental brain research. Experimentelle Hirnforschung. Experimentation cerebrale, 47(3), 329-342. doi:10.1007/BF00239352

Povinelli, D. J. (1987). Monkeys, Apes, Mirrors and Minds: The Evolution of Self-Awareness in Primates. Human Evolution, 2(6), 493-509. doi:10.1007/BF02437424

Rilling, J.K., Barks, S.K., Parr, L.A., Preuss, T.M., Faber, T.L., Pagnoni, G., Bremner, J.D., Votaw, J.R., (2007). A comparison of resting-state brain activity in humans and chimpanzees. Proceedings of the National Academy of Sciences of the United States of America, 104(43), 17146-17151. doi:0705132104 [pii] 10.1073/pnas.0705132104 [doi]

Sackett, G. P. (1966). Monkeys reared in isolation with pictures as visual input: evidence for an innate releasing mechanism. Science, 154(755), 1468-1473. Retrieved from http://www.ncbi.nlm.nih.gov/entrez/query.fcgi? $\mathrm{cmd}=$ retrieve $\& \mathrm{db}=$ pubmed $\&$ dopt $=$ abstract $\&$ list_uids $=4958618$ 
Schweinberger, S. R., Pfütze, E.-M., \& Sommer, W. (1995). Repetition priming and associative priming of face recognition: Evidence from event-related potentials. Journal of Experimental Psychology: Learning, Memory, and Cognition. doi:10.1037/0278-7393.21.3.722

Sui, J., Zhu, Y., \& Han, S. (2006). Self-face recognition in attended and unattended conditions: an event-related brain potential study. NeuroReport, 17(4), 423-427. Retrieved from http://www.ncbi.nlm.nih.gov/pubmed/16514370

Taglialatela, J. P., Russell, J. L., Schaeffer, J. A., \& Hopkins, W. D. (2008). Communicative signaling activates “Broca"s' homolog in chimpanzees. Current Biology, 18(5), 343-348. doi:S0960-9822(08)00096-1 [pii] 10.1016/j.cub.2008.01.049

Taglialatela, J. P., Russell, J. L., Schaeffer, J. A., \& Hopkins, W. D. (2009). Visualizing vocal perception in the chimpanzee brain. Cerebral Cortex, 19(5), 1151-1157. doi:bhn157 [pii] 10.1093/cercor/bhn157

Tallon-Baudry, C., \& Bertrand, O. (1999). Oscillatory gamma activity in humans and its role in object representation. Trends in Cognitive Sciences, 3(4), 151-162. doi:10.1016/S1364-6613(99)01299-1

Tomonaga, M. (2007). Visual search for orientation of faces by a chimpanzee (Pan troglodytes): face-specific upright superiority and the role of facial configural properties. Primates, 48(1), 1-12. doi:10.1007/s10329-006-0011-4 [doi]

Tomonaga, M., Itakura, S., \& Matsuzawa, T. (1993). Superiority of conspecific faces and reduced inversion effect in face perception by a chimpanzee. Folia Primatologica, 61(2), 110-114.

Tsao, D. Y., Freiwald, W. A., Knutsen, T. A., Mandeville, J. B., \& Tootell, R. B. H. (2003). Faces and objects in macaque cerebral cortex. Nature neuroscience, 6(9), 989-995. doi:10.1038/nn1111

Ueno, A., Hirata, S., Fuwa, K., Sugama, K., Kusunoki, K., Matsuda, G., Fukushima, H., Hiraki, K., Tomonaga, M., Hasegawa, T. (2008). Auditory ERPs to stimulus deviance in an awake chimpanzee (Pan troglodytes): towards hominid cognitive neurosciences. PLoS ONE, 3(1), e1442. doi:10.1371/journal.pone.0001442 [doi]

Ueno, A., Hirata, S., Fuwa, K., Sugama, K., Kusunoki, K., Matsuda, G., Fukushima, H., Hiraki, K., Tomonaga, M., Hasegawa, T. (2010). Brain activity in an awake chimpanzee in response to the sound of her own name. Biology letters, 6(3), 311-3. doi:10.1098/rsbl.2009.0864 


\section{Acknowledgments}

479 This study was supported by the Center for Evolutionary Cognitive Science at The University of

480 Tokyo. 


\section{Figure 1}

Figure 1

Stimulus images used in the present study. In Experiment 1, the subject was presented seven images of chimpanzee faces in the three categories of unfamiliar, familiar, and selfimages. In Experiment 2, the subject was presented six images of human faces with unfamiliar and familiar categories. Note that only one example of each category is shown for Experiment 2 for privacy protection.

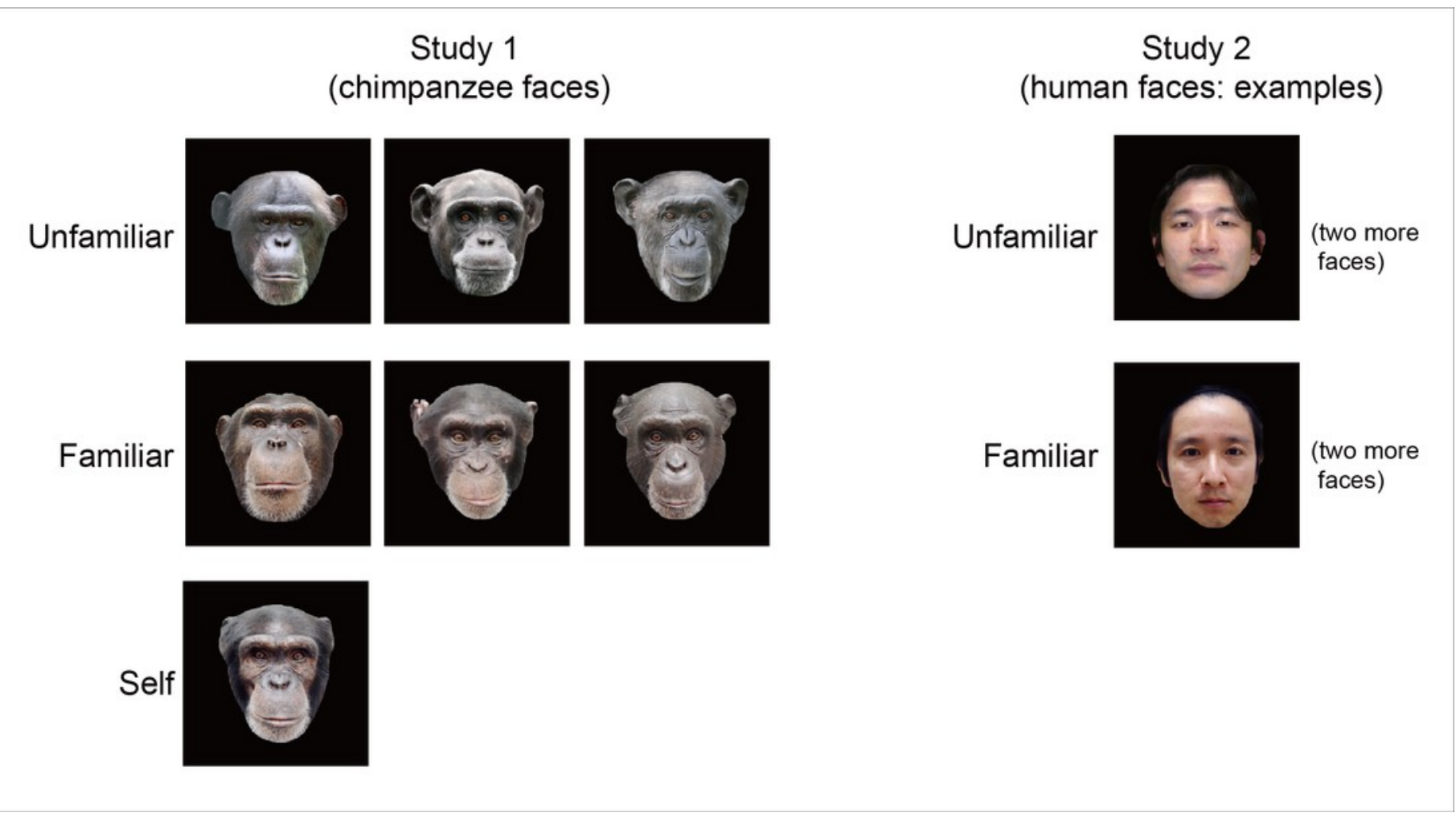




\section{Figure 2}

Figure 2

Averaged ERP waveforms elicited by images of unfamiliar and familiar conspecific faces as well as the subject's own face in Experiment 1. The blue-shaded squares overlaid on the waveforms show the periods of statistically significant main effects of stimulus category. The solid lines below the waveforms show the periods of significant difference between unfamiliar and familiar (green) and unfamiliar and self (orange) images.
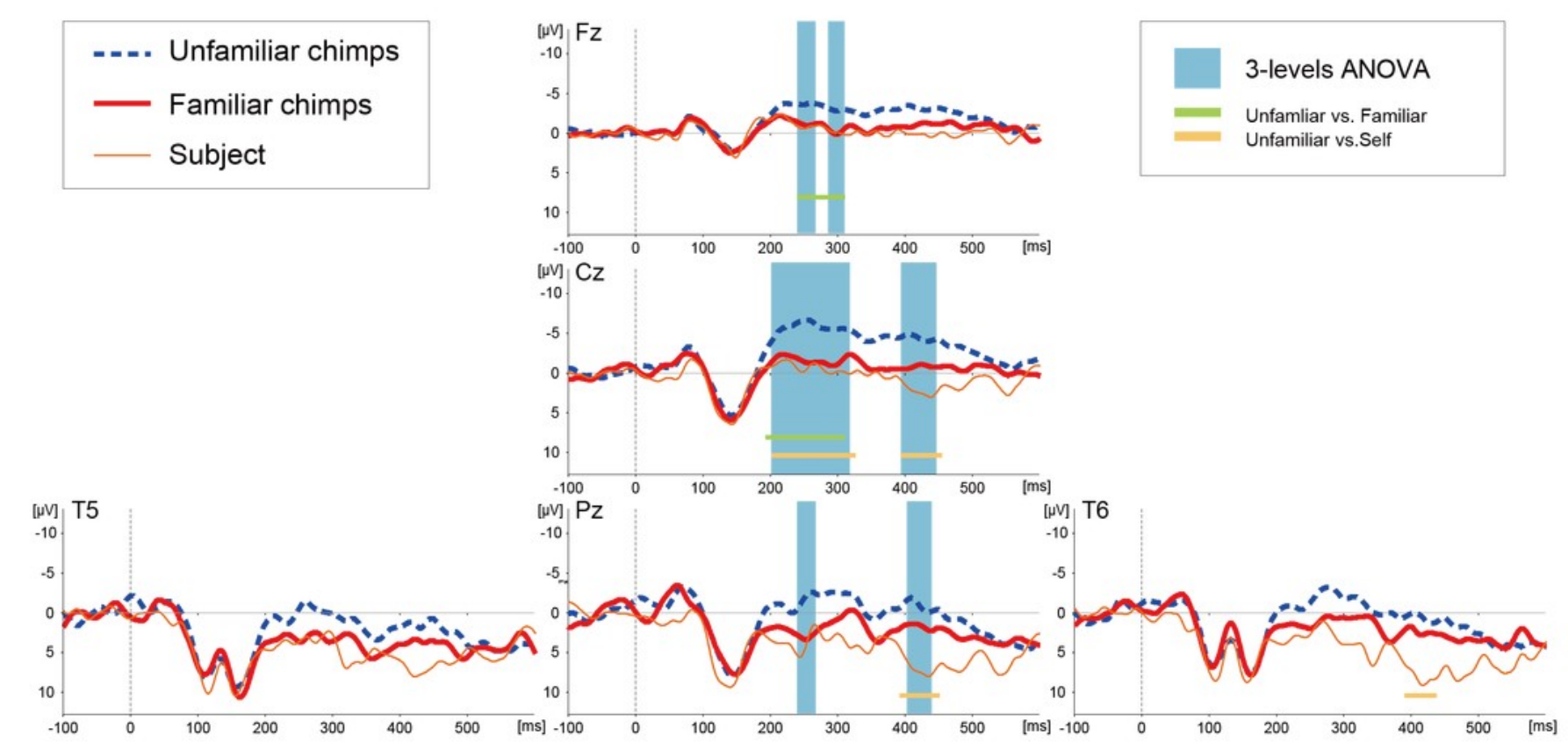


\section{Figure 3}

Figure 3

Averaged ERP waveforms elicited by images of unfamiliar and familiar human faces in Experiment 2.

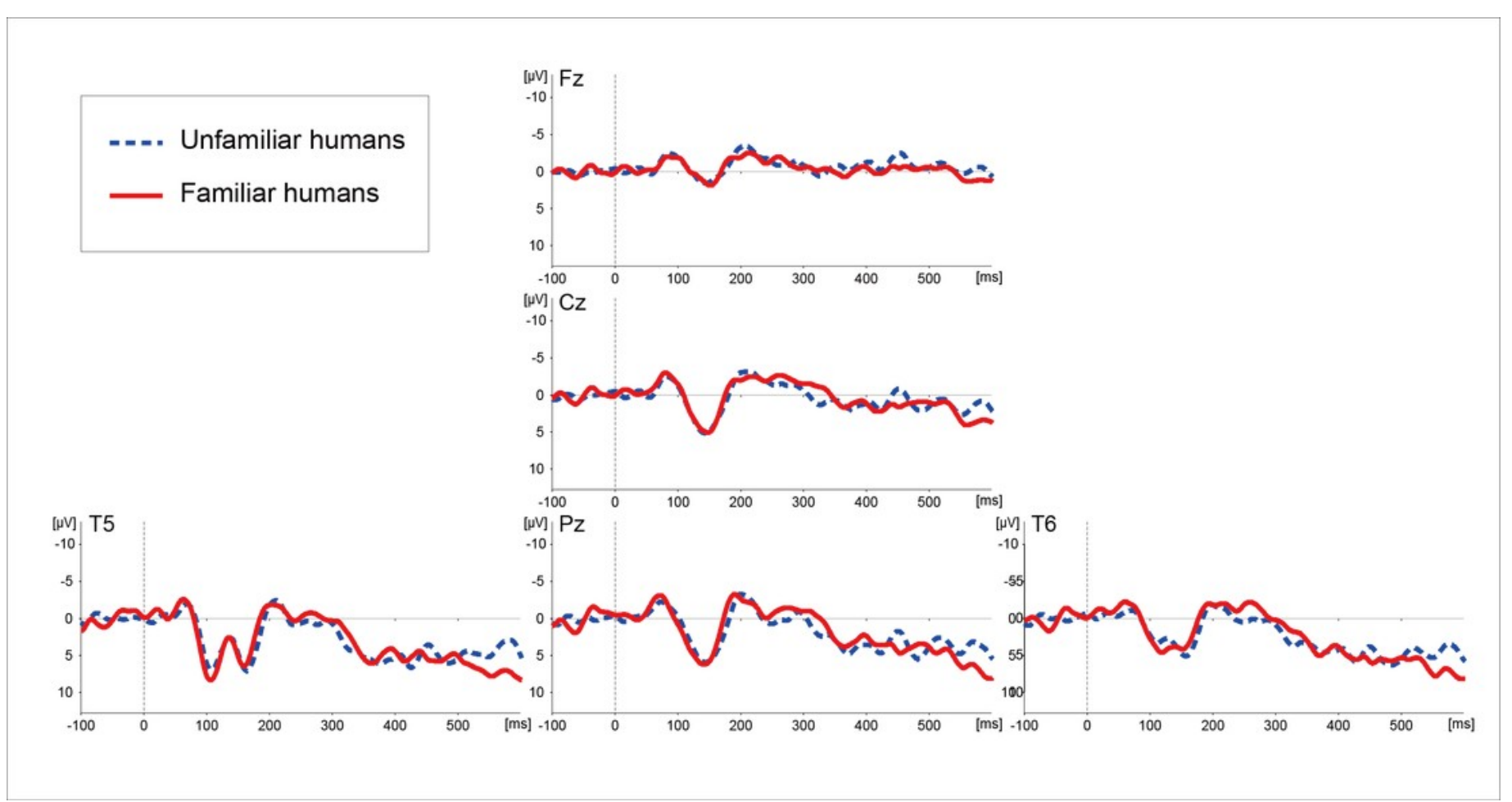




\section{Table 1 (on next page)}

\section{Table 1}

Time ranges where ERPs for each category differed significantly in Experiment 1 (post stimulus latencies in $\mathrm{ms})$. 
Test

Electrodes Time ranges of statistical significance

$\begin{array}{lll}\text { 3-levels ANOVA } & \text { Fz } & 232-269,286-309 \\ \text { Cz } & 201-317,393-445 \\ & \text { Pz } & 232-269,402-438 \\ & & \\ \text { Unfamiliar vs. Familiar } & \text { Fz } & 232-310 \\ & \text { Cz } & 192-309 \\ \text { Unfamiliar vs. Self } & & \\ & & \\ & \text { Cz } & 201-325,392-453 \\ & \text { T6 } & 396-453\end{array}$

